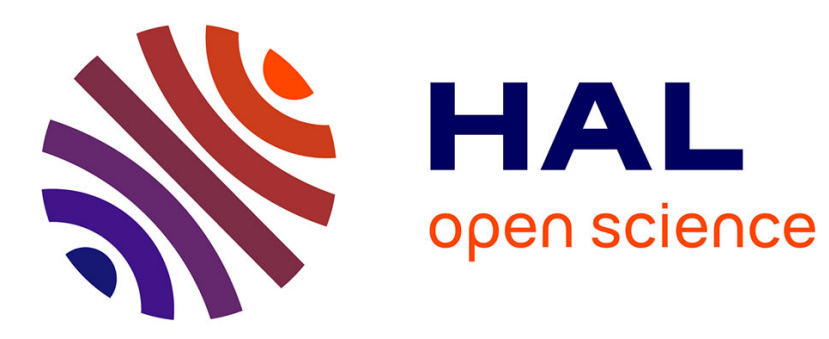

\title{
Couches minces supraconductrices
}

\author{
H.A. Combet, E. Guyon, J. Maldy
}

\section{To cite this version:}

H.A. Combet, E. Guyon, J. Maldy. Couches minces supraconductrices. Revue de Physique Appliquée, 1969, 4 (4), pp.569-573. 10.1051/rphysap:0196900404056900 . jpa-00243334

\section{HAL Id: jpa-00243334 https://hal.science/jpa-00243334}

Submitted on 1 Jan 1969

HAL is a multi-disciplinary open access archive for the deposit and dissemination of scientific research documents, whether they are published or not. The documents may come from teaching and research institutions in France or abroad, or from public or private research centers.
L'archive ouverte pluridisciplinaire HAL, est destinée au dépôt et à la diffusion de documents scientifiques de niveau recherche, publiés ou non, émanant des établissements d'enseignement et de recherche français ou étrangers, des laboratoires publics ou privés. 


\title{
GOUGHES MINGES SUPRACONDUGTRICES
}

\author{
H. A. COMBET, E. GUYON et J. MALDY.
}

Introduction. - Le plan de discussion proposé aux participants était le suivant :

- Techniques de préparation des couches.

- Propriétés physiques à l'état normal et à l'état supraconducteur.

- Techniques de mesure sur les couches.

- Effet tunnel et effet Josephson.

- Effets de proximité.

Nous avons présenté dans le tableau I les techniques possibles de préparation des couches minces en indiquant éventuellement les travaux connus, actuels ou envisagés, les utilisant. Cette méthode de présentation, peut-être arbitraire, devrait néanmoins faciliter le contact entre groupes ayant des problèmes technologiques analogues. Les références citées dans ce tableau sont loin d'être exhaustives et l'on peut déjà trouver des comptes rendus de travaux récents dans les références [1] et [2].

Problèmes de pureté et de structure des couches. Une étude générale de l'influence des paramètres de dépôt sur la croissance et l'état structural des couches minces est faite dans la référence [18]. Nous nous sommes bornés à en résumer les principaux effets dans le tableau II.

L'orientation privilégiée éventuelle des couches peut influer sur leurs propriétés supraconductrices (anisotropie de la vitesse de Fermi, de la bande interdite...). Or, si les possibilités de texture des couches évaporées sur supports amorphes sont connues depuis un certain temps [22], il n'en va pas de même pour les couches obtenues par pulvérisation cathodique.

L'effort de corrélation entre propriétés structurales et supraconductrices des couches minces a été peu entrepris jusqu'à maintenant, sauf pour des couches de plomb (étude des forces d'ancrage [23]) et peutêtre de niobium (pour des cavités hyperfréquences [6]).

Les problèmes de mesure in situ de la pureté des couches ou d'étude de leurs défauts structuraux sans introduction d' « artefacts » se posent comme pour les couches minces non supraconductrices.

Techniques de mesure. - Les températures et champs critiques sont obtenus [7], [24], [25] à partir de mesures de résistivité, de susceptibilité dynamique (Physique des Solides, Orsay), et donnent des résultats en accord avec les théories existantes [26-28]. Des mesures d'impédance de surface et d'aimantation sont en cours de mise au point [29]. Par contre, les mesures de conductivité thermique qui sont cependant bien adaptées à l'étude des films [30] ne sont pas faites en France. Les deux dernières techniques mesurent des propriétés de la « masse » du film, alors que les mesures résistives sont très souvent influencées par l'effet des bords des films ou d'inhomogénéités locales. L'impédance de surface donne des résultats comparables à ceux de l'effet tunnel.

Mémoires supraconductrices. - Le problème des mémoires supraconductrices n'a pas été abordé pendant les discussions. Leur développement n'est limité, de l'avis général, que par les difficultés technologiques inhérentes à la miniaturisation et à l'intégration de ces mémoires et non par des phénomènes plus fondamentaux. Nous pouvons citer néanmoins les projets de la Société Alcatel :

- 1969 : $10^{5}$ E.B. (7 500 E.B. $/ \mathrm{cm}^{2}$ ) avec adresse intégrée.

- 1970: $6 \times 10^{6}$ E.B.

- Coût espéré : 0,1 centime/E.B.

Concurremment à ces mémoires à grande capacité et si elles débouchent dans le public, se posera le problème de la réalisation de logiques et de mémoires supraconductrices très rapides, de faible capacité. Une solution pourrait être apportée par les cryotrons à effet Josephson [3].

La possibilité existe aussi d'éléments de mémoires utilisant des effets de proximité entre films ferromagnétiques et supraconducteurs [4], ]5].

L'avantage de tels éléments est que l'information mise en mémoire n'est pas détruite par un réchauffement éventuel au-dessus de la température critique des supraconducteurs.

Effet tunnel et effet Josephson. - L'effet tunnel est un moyen très puissant d'étude des supraconducteurs. Il donne en particulier une mesure de la densité d'états dont découle l'ensemble des propriétés de transport supraconductrices.

Nous renvoyons aux références [26], [31] et [32] pour la théorie de cet effet. Les comptes rendus de certains travaux expérimentaux récents se trouvent dans [33]. Le groupe d'Orsay s'est particulièrement 
TABLEAU I

\section{Teghniques DE PRÉPARATION DES GOUGHes minces SUPRAGONDUGTRIGeS}

\section{TECHNIQUE}

I. - Evaporation sous vide :

Normale ou ultravide

Bombardement

électronique

Multisource

Flash

II. - Pulvérisation cathodique [12] :

Décharge lumineuse

Réactive

Basse pression

Alternative

ou polarisée

Canon à ions

III. -- Électrolyse :

\footnotetext{
(sur substrat métallique)

V. - Décomposition en phase vapeur
}

IV. - Diffision
Métaux et alliages

\section{MATÉRIAUX}

Matériaux à tension de vapeur élevée

$$
\text { (In, } \mathrm{Pb}, \mathrm{Sn} . . \text { ) }
$$

Matériaux réfractaires ( $\mathrm{Nb} . .$. Alliages, composés

Nitrures

Tous métaux réactifs

$\mathrm{Pb}$

$\mathrm{Nb}$

Composés $A_{3} B$

$\mathrm{Nb}_{3} \mathrm{Sn}$

$\mathrm{Nb}$
OBSERVATIONS

Technique classique et limitée.

Réalisation de cavités hyperfréquences en plomb [6].

Fabrication de "ruban " de plomb en continu. Procédé C.G.E. [7].

Aluminium granulaire [8].

Additifs organiques [9].

Problème : Homogénéisation d'alliages à composants ayant des tensions de vapeur très différentes [10].

Procédé Alcatel.

$\mathrm{Nb}_{3} \mathrm{Sn}[11]$ : Problème : désordre des structures obtenues.

Futur : Fabrication de "ruban " de $\mathrm{Pb}-\mathrm{Bi}$ en continu : C.G.E. (application à des cryotrons de puissance).

Problèmes : Pureté du matériau (contamination par les cloches en Méthode verre). Taille des grains obtenus générale (rapports de résistivités faibles).

Avantage : Possibilité de recouvrir des surfaces de forme quelconque.

Nb-N [13] : envisagé C.G.E.

Ternaire : Nb-Ti-N [14].

Ta et $\mathrm{Ta}-\mathrm{N}$ : très étudiés pour la réalisation de capacités [15].

Réalisation de l'isolant de jonctions tunnel.

Procédé Alcatel [6] : cavités hyperfréquences en $\mathrm{Nb}$.

Équipe E.D.F. : réalisation de matériaux à haute résistivité à l'état normal.

[12] Envisagé : $\mathrm{Nb}_{3} \mathrm{Al}_{x} \mathrm{Ge}_{1-x}$ : Alcatel-C.G.E.

Forme évoluée de la pulvérisation.

Possibilités de micro-usinage (pour mémoire). Réalisation de l'isolant de jonctions Josephson : Air Liquide.

[6] Procédé C.S.F. (cavités hyperfréquences). Bains au fluoborate de plomb avec additifs organiques.

[16] Électroformage de cavités hyperfréquences. Expériences analogues en cours de montage à la C.G.E.

Pour mémoire : $\mathrm{Nb}_{3} \mathrm{Sn}$ en grandes longueurs : procédé "General Electric ".

Technique R.C.A. [17].

[6] Procédé C.S.F. (cavités hyperfréquences). Réduction de pentachlorure de niobium à haute température. 
TABLEAU II

\section{PARAMÈTRES INFLUANT SUR LES PROPRIÉTÉS A L'ÉTAT NORMAL OU SUPRACONDUGTEUR} ET LiÉs a LA PURETÉ ET a L'ÉtAT STRUGTURAL DES GOUGHES

1) Vitesse de dépôt :

2) Gaz résiduels :

3) Nature du substrat :

4) Température du substrat :

5) Vitesse de défilement $d u$ substrat :
Couches évaporées : pureté améliorée par une vitesse élevée.

Couches pulvérisées : risque d'échauffement de la cible et du substrat.

Matériaux non réactifs : effet sélectif [19].

Matériaux réactifs (Influence de l'oxygène occlus sur la température critique (exemples) $\{$ du niobium.

tudes sur la pulvérisation du tantale [12], [18].

Dégazage éventuel

Contraintes introduites par une pseudo-épitaxie.

Irrégularités d'épaisseur dues au "gravage " des grains : $\mathrm{Nb}$ sur $\mathrm{Cu}[6]$.

État de la surface Utilisation de sous-couches :

- adhérence des dépôts,

- neutralisation des effets de bord par effet de proximité.

Contraintes introduites par des coefficients de dilatation différents [18], [20].

\author{
Grains plus petits. \\ Refroidir Dépôts métastables [9]. \\ Continuité de couches plus minces $\left({ }^{*}\right)$. \\ Chauffer $\left\{\begin{array}{l}\text { Éviter la contamination : exemple niobium [21]. } \\ \text { Recuit pour coagulation des bords. }\end{array}\right.$
}

Dans le cas de déposition en continu sur de grandes longueurs [7].

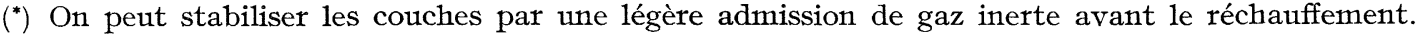

attaché à l'étude des caractéristiques tunnel sous champ magnétique [34-36] et à l'étude des effets de proximité [10], [37]. Le groupe de l'École Normale Supérieure s'est intéressé à la réalisation de barrières tunnel sur de l'aluminium granulaire [8] (vérification de la théorie de Mac Millan [38] reliant la modification du spectre de phonons aux augmentations de Tc, etc.). L'étude des effets de résonance géométrique dans les films purs (effets Tomasch) devrait permettre des études d'interface complémentaires aux mesures d'effets de proximité.

La théorie de l'effet Josephson est exposée en particulier dans les références [26] et [38]. Les nombreuses applications possibles des phénomènes de supraconductivité faible sont passées en revue dans [40]. Si, aux États-Unis, un gros effort a été entrepris pour la réalisation de jonctions Josephson évaporées fiables, on peut noter qu'en France l'Air Liquide commence à étudier la réalisation de couches d'oxydes isolants par pulvérisation réactive à l'aide d'un canon à ions.
Des magnétomètres utilisant des jonctions Josephson évaporées sont en cours d'étude à l'Institut d'Électronique d'Orsay [41].

Nous donnons en tableau III les techniques de préparation des barrières isolantes minces (10 à $50 \AA$ A $)$ utilisées actuellement.

Effets de proximité entre couches supraconductrices et couches non supraconductrices. - Un état supraconducteur peut être induit dans la couche normale si le contact électronique est assez bon. Ces effets ont été particulièrement étudiés théoriquement et expérimentalement par le groupe d'Orsay. Les techniques de mesure employées sont très diverses : mesures de températures et de champs critiques (en continu et en alternatif), mesures par effet tunnel sur des bilames, mesures d'impédances de surface, etc. [42-46]. D'un point de vue fondamental, on en tire en particulier des informations sur l'affinité supraconductrice du métal normal. Toutes ces propriétés (contrôle continu 


\section{TABLEAU III}

1. Oxydation naturelle d'une première électrode métallique $(\mathrm{Al}, \mathrm{Pb}, \mathrm{Nb})$.

2. Oxydation anodique d'une première électrode dans un plasma gazeux. Cette deuxième technique comparable à 1 donne des jonctions plus stables dans le temps et en température ; les paramètres dont dépend l'oxydation sont mieux contrôlés [47].

3. Oxydation complète d’une couche métallique intermédiaire fine ; utilisée avec Al [48].

4. Dépôt d'une couche isolante mince, couche monomoléculaire d'acide gras [49], formvar, SiO, LiF; très délicate.

5. Utilisation de la barrière de Schottky [50] d'un semiconducteur très dopé (Si, GaAs) sur lequel on dépose le film supraconducteur à étudier (on peut aussi appliquer une pointe du semiconducteur sur la couche à étudier). Cette méthode semble très fiable et facilement applicable.

de Tc, champs critiques, supraconductivité induite) n'ont pas encore reçu d'application. Il semble cependant que les mécanismes de proximité jouent un rôle important dans le comportement de supraconducteurs inhomogènes (précipités, systèmes biphasés).

Conclusion. - De nombreuses études ont été menées en France dans le domaine des couches minces supraconductrices; beaucoup d'entre elles à caractère très fondamental et certaines en vue d'applications.

Dans le premier cas, on peut noter que peu de mesures calorimétriques sont faites (chaleur spécifique, conductivité thermique, effet Nernst, etc.). D'autre part, aucune étude n'est entreprise actuellement sur l'émission ou la détection d'hypersons à l'aide de jonctions tunnel.

Dans le deuxième cas, l'étude des propriétés structurales en relation avec les propriétés supraconductrices n'a été entreprise que rarement. Un travail concerté avec les équipes disposant de moyens d'études des structures (contrôle de composition, défauts structuraux, recuits...) est très souhaitable.

Ceci tient en particulier au fait que les propriétés supraconductrices ne dépendent que peu de la structure microscopique détaillée des couches ou d'inhomogénéités locales de tailles inférieures aux longueurs caractéristiques de l'état supraconducteur $\left(10^{2}\right.$ à $10^{4} \AA$ ). Les propriétés thermodynamiques ellesmêmes sont assez indépendantes de l'épaisseur du film. Néanmoins des notions classiques (le libre parcours moyen résiduel, taux de réflexion des électrons sur les surfaces, coefficients de diffusion dans les couches) ont semblé, dans ces discussions, très mal connues. Un travail concerté avec des équipes d'optique, microscopie électronique, rayons $\mathrm{X}$ menant à une véritable métallurgie des couches minces a été unanimement souhaité.

Par ailleurs, on a pensé que les méthodes de pulvérisation cathodique (évaporation de matériaux réfractaires, d'alliage) devraient être développées.

\section{BIBLIOGRAPHIE}

[1] PAscard (H.), Monographie D.R.M.E., 1968, Propriétés électromagnétiques des couches minces métalliques.

[2] J. Physique. C. R. Coll. sur les Propriétés Électroniques des Couches Minces, Orsay, 22-23 juin 1967, J. Physique, 1968, 29, C2.

[3] Matisoo (J.), Proc. I.E.E.E., 1967, 55, 178.

[4] Meunier (F.) et al. [2].

[5] Deutscher (G.) et Meunier (F.), Conférence Intermag, 1969, Amsterdam (à paraître).

[6] SalaüN (M.), Thèse d'Ingénieur-Docteur, Institut d'Électronique d'Orsay, 24 mars 1969.

[7] MaIDY (J.), voir [2] et Contrat D.G.R.S.T., 1966, $65,34,158$.

[8] KLEIN (J.) et LÉGER (A.), Phys. Lett., 1968, 28 A, 134. LÉGER (A.) et KLEIN (J.), Phys. Lett., 1969, 28 A.

[9] Meunier (F.) et al., Phys. Lett., 1968, 26 A, 309.
[10] MarTine' (A.), Thèse de Doctorat, Service de Physique des Solides, Orsay, 23 novembre 1966.

[11] Hammond (R. H.) et al., 1968, Appl. Supercond. Conf. Gatlinburg, et J. Appl. Phys. (à paraître).

[12] Symposium on the Deposition of Thin Films by Sputtering, Rochester, 9 juin 1966 (U. of Rochester).

[13] Deis (D. W.) et al., 1968, Appl. Supercond. Conf. Gatlinburg, et J.Appl. Phys. (à paraître).

[14] BeLL (H.) et al., J. Appl. Phys., 1968, 39, 2797. ZBASNIK (J.) et al., 1968, Appl. Supercond. Conf. Gatlinburg, et $J$. Appl. Phys. (à paraître).

[15] Voir une bibliographie complète dans [12] et dans Coyne (H. J.) et Tauber (R. N.), J. Appl. Phys., 1968, 39, 5585.

[16] Meyerhoff (R. W.), 1968, Appl. Supercond. Conf. Gatlinburg, et $J$. Appl. Phys. (à paraître).

[17] R.C.A. Review, 1964, 25. 
[18] Francombe (M. H.) dans " Problèmes fondamentaux de la physique des couches minces", Comptes rendus du Symposium de Clausthal-Göttingen, septembre 1965, Niedermayer et Mayer (Vandenbrock et Ruprecht, Göttingen).

[19] CASWeli, (H. L.) et al. [18].

[20] Friday (B. W.) et Mundy (J. I.), 1968, App1. Supercond. Conf. Gatlinburg, et J. Appl. Phys. (à paraître).

[21] Mullen (L. O.) et Sulimivan (D. B.), ibid.

[22] Gandais (M.), Rev. Optique, 1961, 40, 464.

[23] Maldy (J.), Ve Colloque sur la Suprafluidité et la Supraconductivité, Bakouriani, février 1968.

[24] Guyon (E.) et al., J. Physique, 1964, 25, 683.

[25] Maldy (J.) et al., Phys. Lett., 1967, 25 A, 318. MALDy (J.) et al., C. R. Acad.Sci. Paris, 1967, 264 B, 416.

[26] DE GENNES (P. G.), "Superconductivity of Metals and Alloys " (W. A. Benjamin Inc., New York, 1966).

[27] Hurault (J. P.), J. Phys. Chem. Solids., 1968, 29, 1765.

[28] Burger (J. P.) et SaInT-James (D.), dans "Superconductivity ", P. D. Parks (Marcel Dekker Inc., New York) (à paraître).

[29] Pinel, (J. L.), Thèse de $3^{\mathrm{e}}$ cycle, I.N.S.A., Rennes, 1969.

[30] Deutscher (G.) et al., Proceedings of the Intern. Conf. on Low Temp. Phys., Saint Andrews, 1968.

[31] SCHRIEFFER (J. R.), "Theory of Superconductivity "(W. A. Benjamin Inc., New York, 1964).

[32] MAC MrLIAN (W. L.) et Rowel, (J. M.), dans "Superconductivity ", P. D. Parks (Marcel Dekker Inc., New York) (à paraître).
[33] Proceedings of the Intern. Conf. on Low Temp Phys., Saint Andrews, 1968, tome II.

[34] Guyon (E.), Advances in Physics, 1966, 15, 417.

[35] Guyon (E.) et al., Phys. Rev., 1967, 156, 452.

[36] NEDEllec (P.), Thèse de $3^{\text {e }}$ cycle, Service de Physique du Solide, Orsay, 1969.

[37] Mauro (S.), Thèse de $3^{\text {e }}$ cycle, Service de Physique du Solide, Orsay, 1967.

[38] Mac Millan (W. L.), Phys. Rev., 1968, 167, 331.

[39] Libchaber (J.), Rev. Phys. Appl., ce numéro.

[40] Taylor (B. N.), J. Appl. Phys., 1968, 39, 2490.

[41] SAUZAdE (M.), Communication privée.

[42] DE Gennes (P. G.), Rev. Mod. Phys., 1964, 36, 225.

[43] Deutscher (G.) et DE Gennes (P. G.), dans «Superconductivity ", P. D. Parks (Marcel Dekker Inc., New York) (à paraître).

[44] Huraulit (J. P.), Thèse de Doctorat, Service de Physique du Solide, Orsay, 29 février 1968.

[45] Rosennlatit (J.) et al., Proc. of the Intern. Conf. on Low Temp. Phys., Saint Andrews, 1968.

[46] Burger (J. P.), Cours d'été franco-allemand, Saarbrücken, septembre 1968.

[47] Mires (J.) et SMiTH (P.), J. Electrochem. Soc., 1963, 110, 1240.

[48] TAylor (B. N.), Thèse, 1964, U. of Pennsylvania, Philadelphia. Ce travail est une excellente référence des effets tunnel entre supraconducteurs.

[49] Mrress (J. C.) et McMahon (H. O.), J. Appl. Phys., 1961, 32, 1176.

[50] Rowell (J. M.) et ChyNoweth (A. G.), Bull. Am. Phys. Soc., 1962, 7, 472. 